\title{
Ingestion and selection of suprabenthic crustaceans by small-sized fishes in a lower saltmarsh system
}

\author{
Yoko Wakabara, Maurea Nicoletti Flynn \& Airton Santo Tararam \\ Instituto Oceanográfico da Universidade de São Paulo \\ (Caixa Postal 66149, 05315-970, São Paulo, SP, Brasil)
}

\begin{abstract}
- Abstract: This study was performed in the lower saltmarsh system of the Arrozal, in the Cananéia lagoon estuarine region ( $\left.25^{\circ} 02^{\prime} \mathrm{S}-47^{\circ} 56^{\circ} \mathrm{W}\right)$, Brazil. Suprabenthic fauna was surveyed with a small sledge and fishes were captured with casting and set nets to analyse: crustacean fauna as food for local fish species; difference in the diet at different times of the year; if there is diet overlap between species and the feeding behaviour of the species analysed. The fauna of Arrozal is poor in species, dominated mainly by Metamysidopsis elongata atlantica, Acartia lilljeborgi, Atylus minikoi, decapod larvae, and reveals a strong seasonal variation. The fishes were all carnivorous with suprabenthic crustacean as their main food resource. Seasonal changes in food supply are also reflected in the diet. Of the 12 fish species collected six were opportunistic feeders whereas six others were selective feeders. Food overlap value of 0.08 for all of the fish community indicates an almost completely distinct food niches. The increased overlapping of summer food between Cathorops spixii and species of Group II and between Oligoplites sp and species of Group I seems to have two different explanations: 1) the minnishing of food supply for species feeding on benthic originated suprabenthic crustaceans and 2) overabundance of planktonic forms of suprabenthos as well as a period of high feeding activity of fishes with such diet.
\end{abstract}

- Resumo: $\mathrm{O}$ presente estudo foi realizado no infralitoral contíguo à marisma, na Ponta do Arrozal, região estuarina lagunar de Cananéia $\left(25^{\circ} 02^{\prime} \mathrm{S}-47^{\circ} 56^{\prime} \mathrm{W}\right)$, Brasil. A fauna suprabêntica foi amostrada com uma pequena draga e os peixes capturados com tarrafa e rede de espera, com a finalidade de analisar: a composição de espécies dos crustáceos suprabênticos como itens alimentares dos peixes; diferenças na dieta em diferentes épocas do ano; se ocorre sobreposiçāo alimentar entre as espécies e o comportamento álimentar das espécies de peixe analisadas. A fauna suprabêntica do Arrozal é pobre em espécies, dominada principalmente por Metamysidopsis elongata atlantica, Acartia lillieborgi, Atylus minikoi e larvas de decapoda, e revela uma forte variação temporal. Os peixes são todos carnívoros e têm crustáceos suprabênticos como sua principal fonte alimentar. Variação temporal no suprimento alimentar se reflete na dieta. Das 12 espécies de peixes coletadas, 6 são oportunistas e 6 seletivas, em relação à captura do alimento. $O$ valor da sobreposição alimentar de 0,08 para toda a comunidade de peixes indica nichos alimentares completamente distintos. $O$ aumento de sobreposição alimentar no verão entre Cathoropsis spixii e espécies do Grupo II e Oligoplites sp e espécies do Grupo I parece ter duas explicações diferentes: 1) a diminuição do fornecimento alimentar para as espécies que se alimentaram de crustáceos suprabênticos com origem bêntica e 2) superabundância de formas planctônicas do suprabentos, assim como um período de alta atividade alimentar para peixes com esta dieta.

- Descriptors: Suprabenthic crustaceans, Fish food, Niche overlap, Southeastern Brazil.

- Descritores: Crustáceos suprabênticos, Alimento de peixes, Sobreposição alimentar, Sudeste do Brasil.

\section{Introduction}

In estuaries, density and biomass of permanent suprabenthos, the same as hyperbenthos (Sibert, 1981;

Contr. no. 799 do Inst. Oceanogr. da Usp.
Mees \& Hamerlynck, 1992 and Mees et al., 1993), are much higher than in neighboring coastal areas (Mees \& Hamerlynck, op. cit.). The importance of suprabenthic small crustaceans in the diets of estuarine fishes is well established and has been the subject of several investigations (Chao \& Musick, 1977; Sibert, op. cit.). 
According to Sibert (1981), suprabenthic populations are composed of surface dwelling benthic and planktonic species presenting upward and downward movements respectively. Components of both origins are found in the diet of small fish inhabiting the infralittoral adjacent to the lower marsh at Arrozal, Cananéia lagoon estuarine region, and suprabenthic crustaceans comprises more than $70 \%$ of the diet of all fishes considered (Wakabara et al., 1993).

The feeding ecology of small sized fish has increasingly become the subject of investigation (Zander, 1979; Evans, 1984; Pihl, 1985; Thorman \& Wiederholm, 1983 and 1986). These studies proved the importance of the food supply, competition with related species, availability of the prey organisms and the influence of the predatory activity as influencing food choice. Nevertheless, much of the prey selectivity has not yet been explained.

Our primary objectives were to describe suprabenthic crustacean food resources utilized by fish species; to determine whether differences or overlap in diet occurred between species and at different times of the year, and to evaluate whether the diet composition is influenced by the presence and abundance of the prey items only or whether in addition, it is affected by a selective behaviour.

\section{Material and methods}

This study was conducted in Cananéia lagoon estuarine region (2502'S-47 $56^{\prime} \mathrm{W}$ ) São Paulo (Fig. 1). The material was collected monthly from August, 1981 to July, 1982 at the infralittoral of Arrozal, 1-3 m depth, where plants of Spartina altemiflora (Loiseleur), appear in a low density. The suprabenthic crustacean fauna was surveyed with a small sledge. The sampling gear consisted of a steel framework weighting about $5.5 \mathrm{~kg}$ and a nylon bag with $0.5 \mathrm{~mm}$ stretch mesh fixed from the inside. Fifteen-minutes' tows were made at each $15 \mathrm{~m}$ long transect, perpendicularly to the water line. Six transects were sampled monthly corresponding to approximately $360 \mathrm{~m}^{2}$. Fishes were captured using a $16 \mathrm{~m}$ casting net (height $3 \mathrm{~m}$ and $3 \mathrm{~cm}$ stretch mesh) thrown in the water from a boat (5 times in each month sampled). A $15 \mathrm{~m}$ set net $(1.30 \mathrm{~m}$ height and 2 $\mathrm{cm}$ stretch mesh) was placed in the water for 30 minutes. All fishes were collected at a diurnal high tide and presented low vacuity index besides a good amount of fresh items in the stomachs.

Macrofauna samples were washed through a sieve of $0.5 \mathrm{~mm}$ mesh size and preserved in $70 \%$ ethanol. The fauna was sorted under a binocular microscope and the crustaceans were identified to species, when possible, and counted. We collected 15 groups of crustaceans but only 8 in sufficient numbers for quantitative analyses.
Fishes were preserved in ice boxes and taken to the laboratory, where they were identified, weighted and measured. Stomachs were cut longitudinally under a stereoscopic microscope and crustacean food items were identified to species level, whenever possible, counted and their volume directly measured. In certain occasions the volume of some items was immeasurable. Empty stomachs were recorded for calculation of the vacuity index and then discarded.

For the analyses and presentation of data, monthly samples were grouped as follows: spring (October December); summer (January - March); autumn (April June) and winter (July - September).

For quantitative analyses only fish and crustacean species with at least two specimens in average per sample were considered, according to Jackson (1972). The overall similarity of diets based on volume of each prey item from the different species of fish were measured with the Morisita index (Sokal and Sneath, 1973). Groupings were determined by WPGA (Weighted Pair Group Average) and were arbitrarily associated at similarity coefficients exceeding 0.60 (Davis, 1973).

Feeding preferences (FP) were calculated by the linear index of food selection (Strauss, 1979), when the number of sampled fishes exceded 10 individuals:

$$
\mathrm{Li}=\mathbf{r}-\mathbf{p i}
$$

where $r i$ is the relative abundance of a species in the stomach and $\mathrm{pi}$ is the relative abundance of a species in the natural habitat samples. It ranges from -1 to +1 with positive values indicating preference and négative values indicating avoidance or inaccessibility. The expected value of the index for random feeding is zero: Electixity values were tested by a two-sided Wilcoxon's signed rank test (Kohler \& Ney, 1982).

Diet overlap was measured using the Schoener index (Schoener, 1970). It ranges from 0 (no overlap) to 1 (complete overlap). Overlap in this index is generally considered to be biologically significant when the value exceeds 0.60 (Zaret \& Rand, 1971; Mathur, 1977).

Stomach contents were analyzed for each $\$$ ish species or group of species using the indexes:

1. Numerical abundance $(\mathrm{N})$ - the number of each crustacean prey item in all non empty stomachs.

2. Percentage of the total number (F\%) - the percentage that each food category contributed to the,total number of crustacean food items in all stomachs.

3. Percentage of total volume (V\%) - the percentage that each food category contributed to the total volume of crustacean food items in all stomachs. 
4. Vacuity index (V.I.) - (number of empty stomachs/ total number of stomachs) $\times 100$.
5. Species richness (S.R.) - number of items ingested by a certain fish or group of fishes.

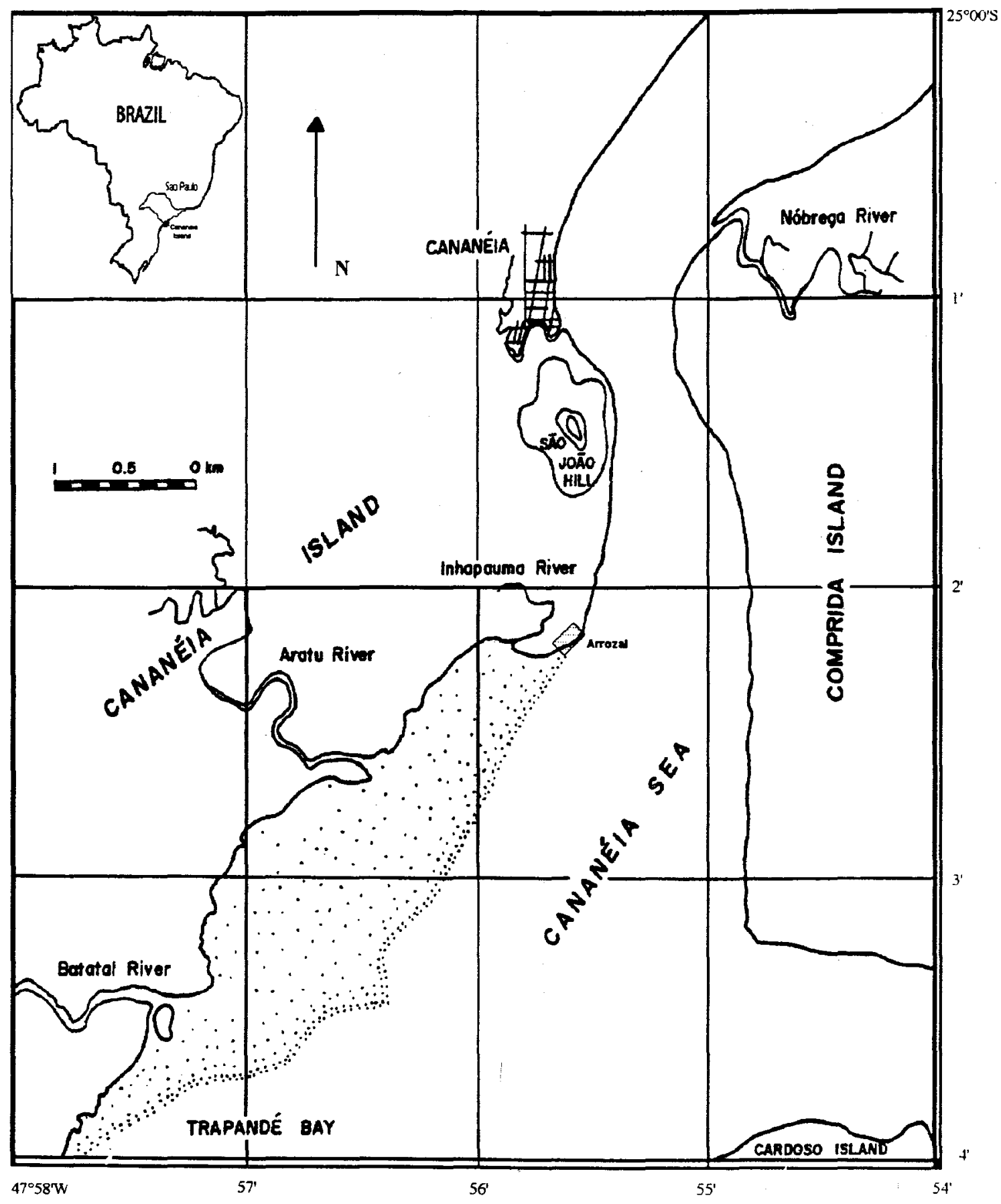

Fig. 1. Location of sampling site (Arrozal) in Cananéia lagoon estuarine region, São Paulo State, southeastern Brazil. 


\section{Results}

Regarding the suprabenthic fauna, a total of 8 crustacean groups were considered among 15 sampled groups for quantitative analyses (Table 1). Crustacean fauna of the suprabenthos at Arrozal ranged in number from 447 specimens in summer to 22,567 in spring. Mysidacea, Copepoda and Amphipoda were the groups with higher species number and abundance among the local suprabenthos. Fluctuations in number of individuals of some species revealed replacement of dominant species and seasonal succession in the area. Metamysidopsis elongata atlantica was preponderant in spring and autumn, Acartia lilljeborgi and decapod larvae in summer, Atylus minikoi and $M$. elongata atlantica in winter.

From the total of 30 collected fish species only 12 could be analyzed numerically. These species are: Stellifer stellifer, Micropogonias furnieri, Oligoplites sp, Cathorops spixii, Isopisthus parvipinnis, Harengula jaguana, Eucinostomus sp, Symphumis jenynsii, Opisthonema oglinum, Anchoviella brevirostris, Haemulon sp, Sphoeroides pachygaster. Number of specimens, number of empty stomachs, total length and weight of fishes considered are in Table 2.

Table 1. Suprabenthic crustacean species sampled during the four seasons of the period (1981-1982) - numerical abundance $(N)$, percentage of the total number (F\%)

\begin{tabular}{|c|c|c|c|c|c|c|c|c|}
\hline \multirow[b]{2}{*}{ SPECIES } & \multicolumn{2}{|c|}{ SPRING } & \multicolumn{2}{|c|}{ SUMMER } & \multicolumn{2}{|c|}{ AUTUMN } & \multicolumn{2}{|c|}{ WINTER } \\
\hline & $\mathrm{N}$ & $\mathrm{F} \%$ & $\mathrm{~N}$ & $\mathrm{~F} \%$ & $N$ & $\mathrm{~F} \%$ & $\mathrm{~N}$ & $\mathrm{~F} \%$ \\
\hline \multicolumn{9}{|l|}{$\overline{\text { OSTRACODA }}$} \\
\hline $\begin{array}{l}\text { Cyprideis salebrosa hartmanni } \\
\text { COPEPODA }\end{array}$ & 45 & 0.199 & 21 & 4.698 & 2 & 0.186 & 154 & 4.419 \\
\hline Acartia liljeborgi & 5 & 0.022 & 279 & 62.416 & 49 & 4.567 & 15 & 0.430 \\
\hline Eucalanus pileatus & - & - & 1 & 0.224 & 2 & 0.186 & 53 & 1.521 \\
\hline Labidocera fluviatilis & 1 & 0.004 & 3 & 0.671 & 24 & 2.237 & 130 & 3.730 \\
\hline Temora stylifera & 7 & 0.031 & 20 & 4.474 & 2 & 0.186 & 34 & 0.976 \\
\hline DECAPODA (larvae) & 16 & 0.071 & 51 & 11.409 & 78 & 7.269 & 21 & 0.603 \\
\hline \multicolumn{9}{|l|}{ PENAEIDAE } \\
\hline Penaeus brasiliensis & 118 & 0.523 & 13 & 2.908 & 6 & 0.559 & - & - \\
\hline \multicolumn{9}{|l|}{ MYSIDACEA } \\
\hline Metamysidopsis elongata atlantica & 22352 & 99.047 & 17 & 3.803 & 824 & 76.794 & 975 & 27.977 \\
\hline Mysidopsis coelhoi & 2 & 0.009 & 28 & 6.264 & 4 & 0.373 & 8 & 0.230 \\
\hline \multicolumn{8}{|l|}{ TANAIDACEA } & 0.115 \\
\hline \multicolumn{8}{|l|}{ ISOPODA } & 2. 181 \\
\hline Munna cananeia & - & - & - & - & - & - & 47 & 1.349 \\
\hline \multicolumn{9}{|l|}{ AMPHIPODA } \\
\hline Ampithoe ramondi & 2 & 0.009 & - & - & - & - & 320 & 9.182 \\
\hline Atylus minikoi & 3 & 0.013 & - & - & - & - & 1484 & 42.582 \\
\hline Corophium acherusicum & - & - & - & - & - & - & 123 & 3.529 \\
\hline Grandidierella bonnieroides & 1 & 0.004 & 5 & 1.119 & 4 & 0.373 & 41 & 1.176 \\
\hline TOTAL & 22567 & 100.00 & 447 & 100.00 & 1073 & 100.00 & 3485 & 100.00 \\
\hline
\end{tabular}


Table 2. Number of specimens $(\mathrm{N})$, number of empty stomachs $(E)$, length and weight of twelve fish species

\begin{tabular}{lcrrr}
\hline \multicolumn{1}{c}{ Species } & N & E & $\begin{array}{c}\text { Total length } \\
\text { (cm) }\end{array}$ & \multicolumn{1}{c}{$\begin{array}{c}\text { Weight } \\
(\mathrm{g})\end{array}$} \\
\hline Anchoviella brevirostris & 29 & 0 & $6.2-11.0$ & $3.0-11.0$ \\
Cathorops spixii & 64 & 5 & $10.0-19.8$ & $12.0-39.5$ \\
Eucinostomus sp. & 31 & 7 & $8.0-13.0$ & $5.0-25.0$ \\
Haemulon sp. & 26 & 10 & $9.0-12.0$ & $9.0-23.0$ \\
Harengula jaguana & 54 & 7 & $10.0-12.0$ & $10.0-20.0$ \\
Isopisthus parvipinnis & 62 & 30 & $10.9-17.2$ & $11.0-48.0$ \\
Micropogonias fumieri & 117 & 13 & $7.0-17.0$ & $4.5-51.0$ \\
Oligoplites sp. & 84 & 26 & $6.7-14.0$ & $3.0-21.0$ \\
Opisthonema oglinum & 30 & 0 & $9.8-12.9$ & $10.0-25.0$ \\
Symphurus jenynsil & 30 & 4 & $6.5-18.0$ & $3.0-49.0$ \\
Sphoeroides pachygaster & 21 & 4 & $7.8-27.0$ & $8.0-238.0$ \\
Stellifer stellifer & 155 & 74 & $0.5-17.0$ & $10.0-30.0$ \\
\hline
\end{tabular}

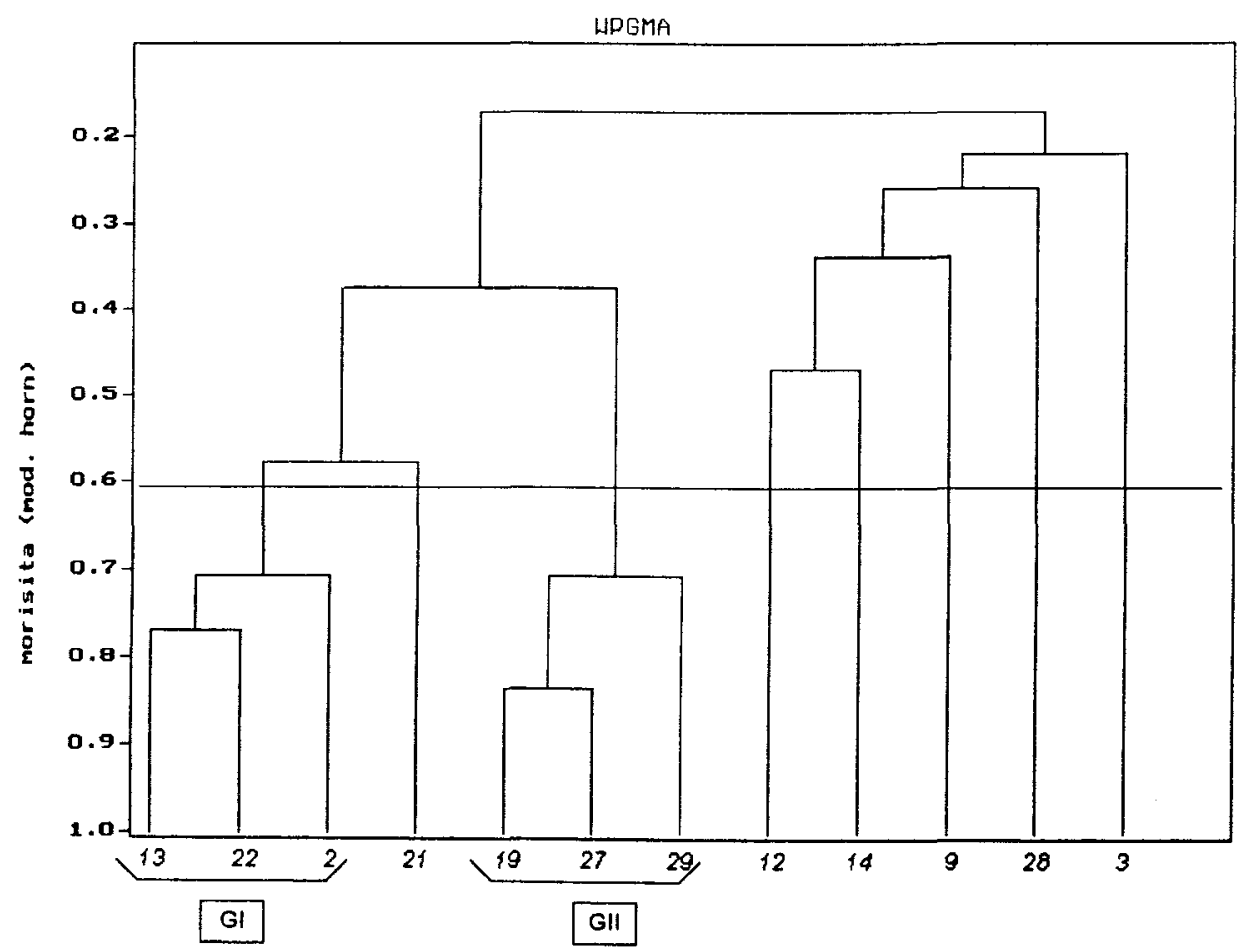

Fig. 2. Cluster analysis based on similarity of food items in the stomach of twelve fish species (Group 1: 2 - Anchoviella brevirostris, 13 - Harengula jaguana, 22 Opistonema oglinum; Group II: 19 - Micropogonias fumieri, 27 - Symphunıs jenynsii, 29- Stellifer stellifer and not grouped species; 3 - Cathorops spixii, 9 - Eucinostomus sp, 12 - Haemulon sp, 14 -Isopisthus parvipinnis, 21 - Oligoplites sp, 28 - Sphoeroides pachygaster).
A cluster analysis was run on trophic data for all 12 fish species (Fig. 2), dividing them into 2 groups with 3 species each. $A$. brevirostris, $H$. jaguana and $O$. oglinum occurred as one group denominated Group I , M. fumieri, $S$. jenynsii and $S$. stellifer occurred as another group denominated Group II. The other species were not grouped.

Species of Group I were not persistent throughout the four seasons (Fig. 3). They decrease in number from spring to summer. In autumn they were absent, presenting maximum number in winter. Their diet comprises 13 species of Copepoda, 5 of Amphipoda and 4 of Mysidacea, besides Ostracoda, Luciferidae, Callianassidae, Decapoda larvae and Tanaidacea (Table 3). Temora stylifera, Labidocera fluviatilis and $M$. elongata atlantica were the most ingested items in spring, $A$. lilljeborgi and decapod larvae in summer, decapod larvae and $L$. fluviatilis in winter. The most rejected or inaccessible items for this group being $M$. elongata atlantica and $A$. minikoi. 
Group 1

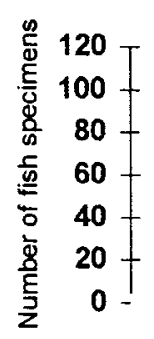

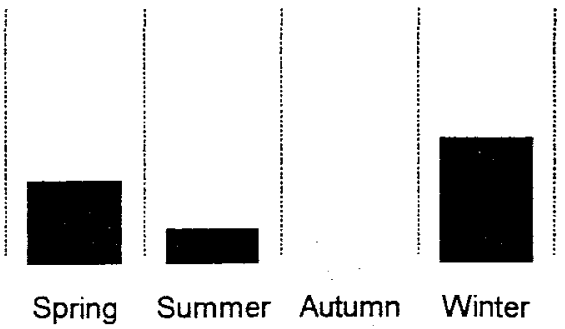

Cathorops spixii

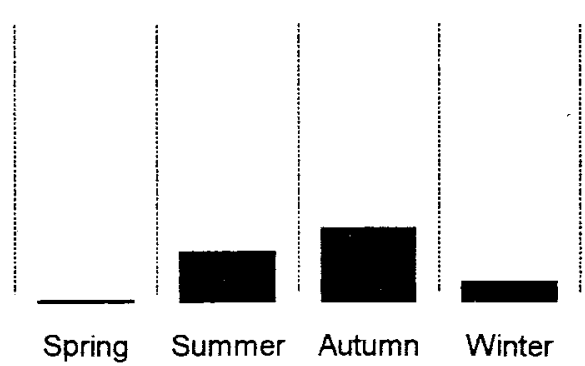

Haemulon sp.
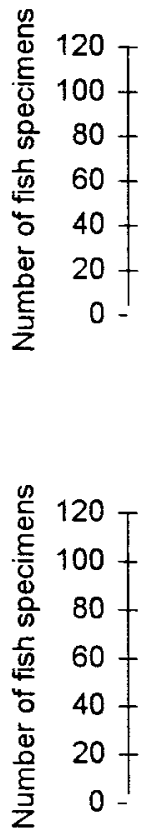
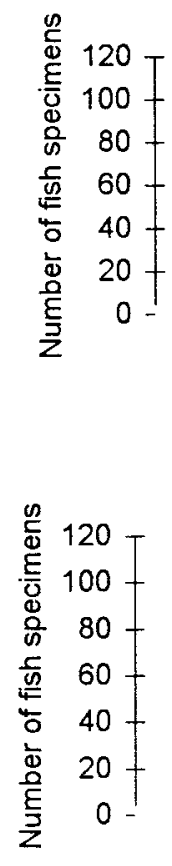

Eucinostomus sp.

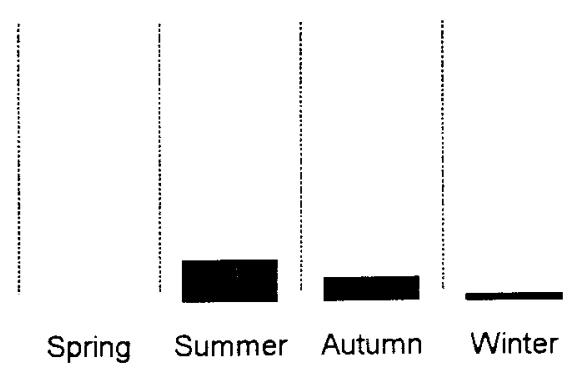

Isopisthus parvipinnis

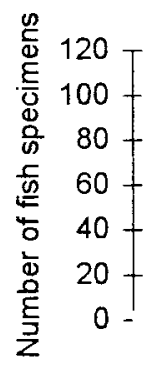

Sphoeroides pachigaster

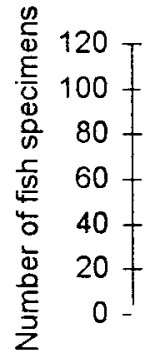

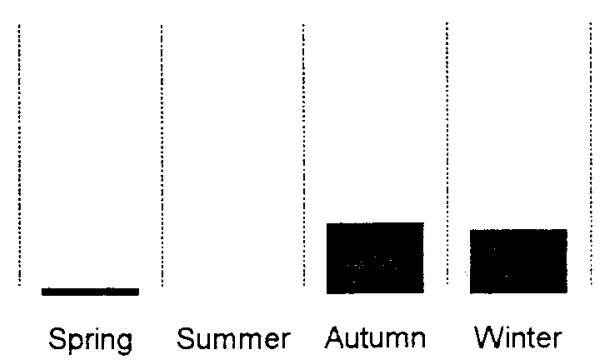

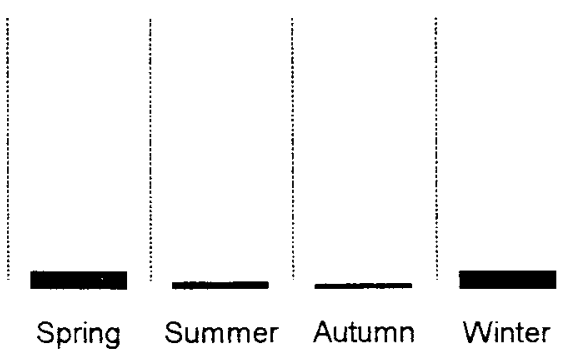

Fig. 3. Abundance of fish species during the period 1981-1982 Gl: Anchoviella brevirostris + Harengula jaguana + Opistonema oglinum Gll: Micropogonias fumieri + Symphums jenynsii + Stellifer stellifer 
Table 3. Crustaceans found in stomachs of Group 1 - Anchoviella brevisrostris $(\mathrm{N}=29)$, Opistonema oglinum $(\mathrm{N}=30)$ and Harengula jaguana $(\mathrm{N}=54)$ - numerical abundance $(\mathrm{N})$, vacuity index (V.I.), species richness (S.R.), feeding preference (FP), percentage of the total number (F\%) and percentage of total volume (V\%)

\begin{tabular}{|c|c|c|c|c|c|c|c|c|c|c|c|c|}
\hline \multirow[b]{3}{*}{ Prey item } & \multicolumn{4}{|c|}{ Spring } & \multicolumn{4}{|c|}{ Summer } & \multicolumn{4}{|c|}{ Winter } \\
\hline & \multicolumn{2}{|c|}{ V.I. $=7.69$} & \multicolumn{2}{|c|}{ S.R. $=16$} & \multicolumn{2}{|c|}{$V .1=25.00$} & \multicolumn{2}{|c|}{ S.R. $=8$} & \multicolumn{4}{|c|}{ V.C. $=0 \quad$ S.R. $=18$} \\
\hline & $\mathrm{F} \%$ & V\% & $\mathrm{FP}$ & $\mathrm{N}$ & $F \%$ & $\vee \%$ & $\mathrm{FP}$ & $\mathrm{N}$ & $\mathrm{F} \%$ & $\mathrm{~V} \%$ & $\mathrm{FP}$ & $\mathrm{N}$ \\
\hline \multicolumn{13}{|l|}{ OSTRACODA } \\
\hline Loxoconcha sp. & - & - & - & - & - & - & - & - & 23.22 & 10.28 & $\underline{0.23}$ & 317 \\
\hline \multicolumn{13}{|l|}{ COPEPODA } \\
\hline Acartia lilljeborgi & 2.28 & 0.99 & 0.00 & 43 & 81.72 & 49.58 & $\underline{0.20}$ & 304 & 17.58 & 7.54 & 0.17 & 240 \\
\hline Centropages velificatus & 0.05 & 0.00 & 0.00 & 1 & - & - & - & - & 0.07 & 0.00 & 0.00 & 1 \\
\hline Corycaeus giesbrechti & - & - & - & - & - & - & - & - & 0.07 & 0.00 & 0.00 & 1 \\
\hline Ctenocalanus vanus s. I. & 0.11 & 0.00 & 0.00 & 2 & - & - & - & - & - & - & - & - \\
\hline Cyclopinidae (not identified) & - & - & - & - & - & - & - & - & 0.15 & 0.00 & 0.00 & 2 \\
\hline Eucalanus pileatus & 0.43 & 0.00 & 0.00 & 8 & 1.07 & 0.84 & 0.01 & 4 & 4.18 & 1.92 & 0.03 & 57 \\
\hline Euterpina acutifrons & - & - & $\because$ & - & 0.81 & 0.00 & 0.00 & 3 & 1.98 & 0.82 & 0.01 & 27 \\
\hline Harpacticoida (not identified) & - & - & - & - & 0.27 & 0.00 & 0.00 & 1 & 1.54 & 1.41 & 0.01 & 21 \\
\hline Labidocera fluviatillis & 14.03 & 13.15 & 0.14 & 264 & 7.26 & 4.20 & 0.07 & 27 & 38.90 & 16.46 & $\underline{0.35}$ & 531 \\
\hline Paracalanus crassirostris & - & - & - & - & - & - & - & - & 0.15 & 0.00 & 0.00 & 2 \\
\hline Pseudodiaptomus acutus & 5.31 & 2.26 & 0.05 & 100 & 5.37 & 3.36 & 0.05 & 20 & 2.56 & 1.09 & 0.02 & 35 \\
\hline Siphonostomatoida (not identified) & 0.05 & 0.00 & 0.00 & 1 & - & - & - & - & - & - & - & - \\
\hline Temora stylifera & 60.36 & 38.33 & $\underline{0.60}$ & 1136 & 0.81 & 0.00 & -0.04 & 3 & 5.27 & 2.19 & 0.04 & 72 \\
\hline DECAPODA (larvae) & 0.53 & - & - & 10 & 2.69 & 42.02 & -0.09 & 10 & 3.44 & 58.29 & 0.02 & 47 \\
\hline \multicolumn{13}{|l|}{ LUCIFERIDAE } \\
\hline Lucifer faxoni & 0.05 & - & - & 1 & - & - & - & - & $\cdot$ & - & - & - \\
\hline \multicolumn{13}{|l|}{ THALASSINIDEA } \\
\hline Callianassidae (not identified) & 0.32 & - & - & 6 & - & $\therefore$ & - & - & - & - & - & - \\
\hline \multicolumn{13}{|l|}{ MYSIDACEA } \\
\hline Bowmaniella brasiliensis & 0.05 & - & - & 1 & - & - & - & - & - & - & - & - \\
\hline Metamysidopsis elongata atiantica & 11.90 & 42.43 & $\underline{-0.88}$ & 224 & - & - & $\cdot$ & - & - & - & $\underline{-0.27}$ & - \\
\hline Mysidopsis coelhoi & 0.05 & 0.00 & 0.00 & 1 & - & - & - & - & 0.07 & 0.00 & 0.00 & 1 \\
\hline Mysidopsis tortonesi & 4.04 & 2.83 & 0.04 & 76 & - & - & - & - & - & - & - & - \\
\hline \multicolumn{13}{|l|}{ TANAIDACEA } \\
\hline Kalliapseudes schubarti & - & - & - & - & - & - & - & - & 0.59 & 0.00 & -0.02 & 8 \\
\hline \multicolumn{13}{|l|}{ AMPHIPODA } \\
\hline Caprella equilibra & 0.43 & 0.00 & 0.00 & 8 & - & - & - & - & - & - & - & - \\
\hline Atylus minikoi & - & - & - & - & - & - & - & - & - & - & -0.42 & - \\
\hline Corophium acherusicum & - & - & - & - & - & - & - & - & 0.07 & 0.00 & -0.03 & 1 \\
\hline Parhyale hawaiensis & - & - & - & - & - & - & - & - & 0.07 & 0.00 & 0.00 & 1 \\
\hline Tiron sp. & - & - & - & - & - & - & - & - & 0.07 & 0.00 & 0.00 & 1 \\
\hline
\end{tabular}


Fishes of Group II were persistent throughout the year and numerically dominant in all seasons. They decreased in number from spring toward summer, increased in autumn and in winter they presented the highest value (Fig. 3). The food spectrum of this group is made up by 34 taxonomic items being Amphipoda, Caridea, Copepoda and Mysidacea with 9, 5, 4 and 3 species respectively the richest food groups in species (Table 4). Mysids were the most ingested items in spring, Alpheus armillatus in summer, $M$. elongata atlantica and $A$. armillatus in autumn and Munna cananeia and Kalliapseudes schubarti in winter. The most rejected or inaccessible items for this groups being $M$. elongata atlantica, $A$. lilljeborgi and $A$. minikoi.

Table 4. Crustaceans found in stomachs of Group II - Stellifer stellifer $(N=155)$, Micropogonias furnieri $(\mathrm{N}=117)$ and Symphurus jenynsii $(\mathrm{N}=30)$ - numerical abundance (N), vacuity index (V.I.), species richness (S.R.), feeding preference (FP), percentage of the total number $(\mathrm{F} \%)$ and percentage of total volume $(\mathrm{N} \%)$

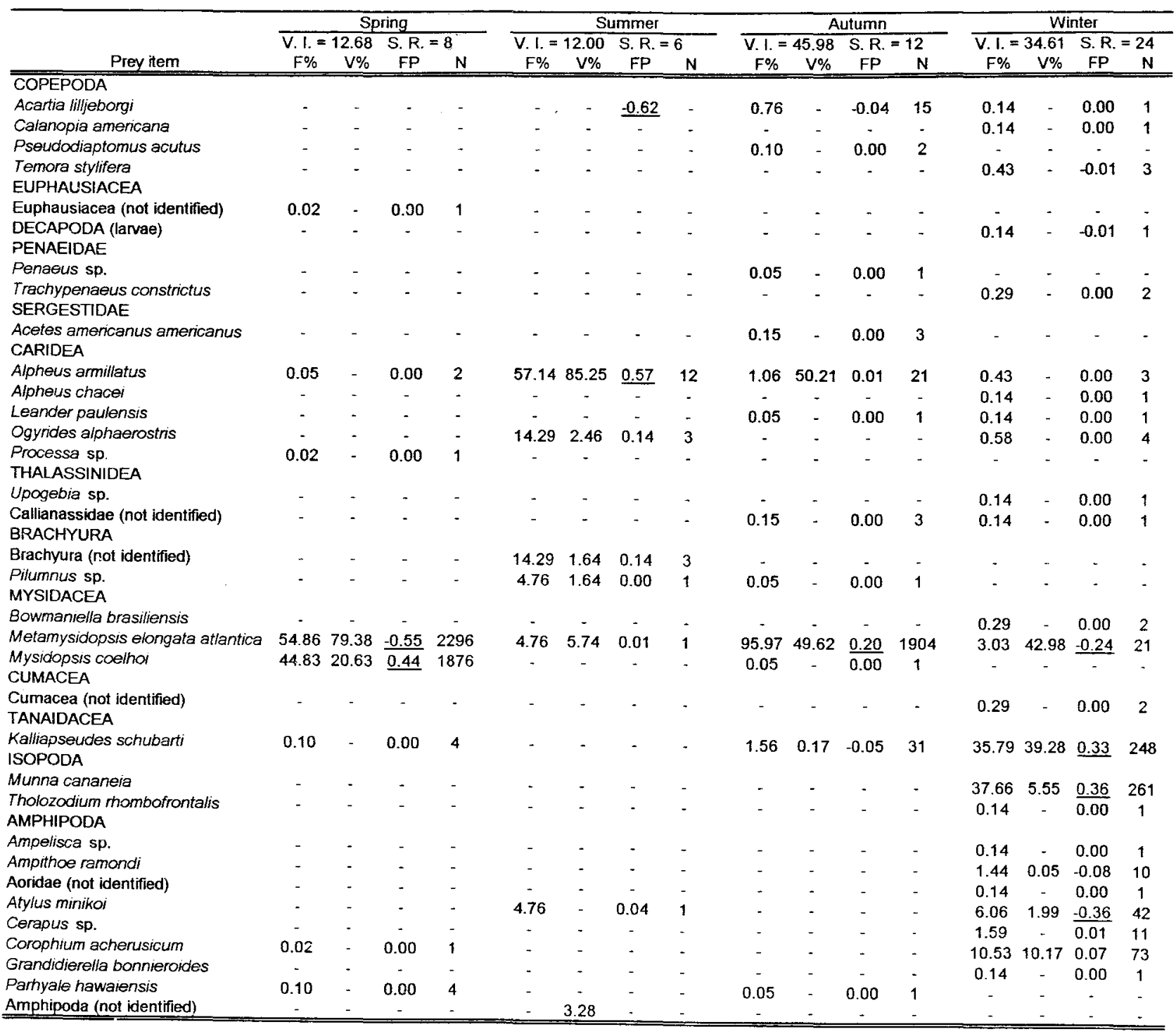


C. spixii presented peak numbers during summer and autumn (Fig. 3). The number of items in the stomach contents varied from 4 , in summer to 14 in autumn (Table 5). The species feeds mainly on $A$. amillatus in summer; Harpacticoidea in autumn; $A$. armillatus and $M$. cananeia in winter. The species showed avoidance to $A$. lilljeborgi and decapod larvae.
Eucinostomus sp was not collected in spring (Fig. 3). In winter it ate no crustacean items. The species had a very narrow food spectrum, with one item in autumn and 2 in summer and the main food item was $K$. schubarti in the two seasons (Table 6). The species showed avoidance to $A$. lilljeborgi and $M$. elongata atlantica.

Table 5. Crustaceans found in stomachs of Cathorops spixii $(N=64)$ - numerical abundance (N), vacuity index (V.I.), species richness (S.R.), feeding preference (FP), percentage of the total number ( $F \%$ ) and percentage of total volume (V\%)

\begin{tabular}{|c|c|c|c|c|c|c|c|c|c|c|c|c|}
\hline \multirow[b]{3}{*}{ Prey item } & \multicolumn{4}{|c|}{ Summer } & \multicolumn{4}{|c|}{ Autumn } & \multicolumn{4}{|c|}{ Winter } \\
\hline & \multicolumn{4}{|c|}{$V .1=4.55 \quad$ S. R. $=4$} & \multicolumn{2}{|c|}{ V.I. $=9.37$} & \multicolumn{2}{|c|}{ S.R. $=14$} & \multicolumn{2}{|c|}{ V.I. $=11.12$} & \multicolumn{2}{|c|}{ S.R. $=7$} \\
\hline & F\% & V\% & $\mathrm{FP}$ & $\mathrm{N}$ & $\mathrm{F} \%$ & $\vee \%$ & FP & $N$ & $\mathrm{~F} \%$ & V\% & FP & $N$ \\
\hline \multicolumn{13}{|l|}{$\overline{\text { COPEPODA }}$} \\
\hline Acartia lilljeborgi & $=$ & $=$ & -0.61 & - & 0.14 & - & -0.04 & 5 & - & - & - & - \\
\hline Cyclopinidae (not identified) & - & - & - & - & 1.52 & - & 0.01 & 54 & - & - & - & - \\
\hline Halicyclops crassicomis & - & $=$ & - & - & 0.23 & - & - & 8 & - & - & - & - \\
\hline Harpacticoida (not identified) & $=$ & $=$ & . & - & 91.49 & 98.00 & $\underline{0.91}$ & 3251 & - & - & - & - \\
\hline Oncaea curta & $=$ & $=$ & - & - & 0.06 & - & - & 2 & - & - & - & - \\
\hline Paracalanus crassirostris & $=$ & $=$ & - & - & 0.14 & - & - & 5 & - & - & - & - \\
\hline Pseudodiaptomus acutus & - & $=$ & - & - & 0.03 & - & - & 1 & - & - & - & - \\
\hline Temora stylifera & - & $=$ & - & - & 0.03 & - & - & 1 & - & - & - & - \\
\hline \multicolumn{13}{|l|}{ EUPHAUSIACEA } \\
\hline Euphausiacea (not identified) & - & $=$ & - & - & 0.17 & - & - & 6 & - & - & - & - \\
\hline DECAPODA (larvae) & - & $=$ & -0.11 & - & - & - & - & - & - & - & - & - \\
\hline \multicolumn{13}{|l|}{ PENAEIDAE } \\
\hline Penaeus sp. (post-larvae) & 8.33 & $=$ & 0.06 & 1 & 0.11 & - & - & 4 & - & - & - & - \\
\hline Trachypenaeus constrictus & 8.33 & $=$ & 0.08 & 1 & - & - & - & - & - & - & - & - \\
\hline \multicolumn{13}{|l|}{ CARIDEA } \\
\hline Alpheus armillatus & 75.00 & 100.00 & 0.75 & 9 & - & - & - & - & 3.57 & 49.67 & 0.04 & 1 \\
\hline Ogyrides alphaerostris & - & - & $=$ & - & 0.03 & - & - & 1 & - & - & - & - \\
\hline \multicolumn{13}{|l|}{ THALASSINIDEA } \\
\hline Callianassidae (not identified) & - & - & - & - & - & - & - & - & 3.57 & - & 0.04 & 1 \\
\hline \multicolumn{13}{|l|}{ MYSIDACEA } \\
\hline \multicolumn{13}{|l|}{ TANAIDACEA } \\
\hline $\begin{array}{l}\text { Kalliapseudes schubarti } \\
\text { ISOPODA }\end{array}$ & \multicolumn{3}{|c|}{ ISOPODA } & - & 2.53 & - & -0.04 & 90 & 17.86 & 8.28 & - & 5 \\
\hline Munna cananeia & - & - & - & - & 3.54 & 2.00 & 0.03 & 126 & 7.14 & 41.39 & -0.05 & 2 \\
\hline \multicolumn{13}{|l|}{ AMPHIPODA } \\
\hline Ampithoe ramondi & - & - & - & - & - & - & - & - & 3.57 & - & 0.00 & 1 \\
\hline Atylus minikoi & - & - & - & - & - & - & - & - & 57.14 & 0.66 & $\underline{0.30}$ & 16 \\
\hline Corophium acherusicum & 8.33 & - & 0.08 & 1 & - & - & - & - & 7.14 & - & 0.06 & 2 \\
\hline Monoculodes nyei & - & - & - & - & 0.03 & - & - & 1 & - & - & - & - \\
\hline \multicolumn{13}{|l|}{ Not Consumed } \\
\hline Metamysidopsis elongata atlantica & - & - & - & - & - & - & - & - & - & - & -0.27 & - \\
\hline
\end{tabular}


Table 6. Crustaceans found in stomachs of Eucinostomus sp $(\mathrm{N}=31)$ - numerical abundance (N), vacuity index (V.I.), species richness (S.R.), feeding preference (FP), percentage of the total number (F\%) and percentage of total volume (N\%)

\begin{tabular}{|c|c|c|c|c|c|c|c|c|}
\hline \multirow[b]{3}{*}{ Prey item } & \multicolumn{4}{|c|}{ Summer } & \multicolumn{4}{|c|}{ Autumn } \\
\hline & \multicolumn{2}{|c|}{$\mathrm{V} . \mathrm{I} .=22.23 \mathrm{~s}$} & \multicolumn{2}{|c|}{ S. R. $=2$} & \multicolumn{2}{|c|}{ V. I. $=30.50$} & \multicolumn{2}{|c|}{ S. R. = 1} \\
\hline & $\mathrm{F} \%$ & V\% & FP & $\mathrm{N}$ & $\mathrm{F} \%$ & $\mathrm{~V} \%$ & $\mathrm{FP}$ & $\mathrm{N}$ \\
\hline \multicolumn{9}{|l|}{ COPEPODA } \\
\hline \multicolumn{9}{|l|}{ PENAEIDAE } \\
\hline Penaeus sp. & 11.11 & 86.96 & 0.08 & 1 & - & - & - & - \\
\hline \multicolumn{9}{|l|}{ MYSIDACEA } \\
\hline \multicolumn{9}{|l|}{ TANAIDACEA } \\
\hline Kalliapseudes schubarti & 88.88 & 13.04 & 0.87 & 8 & 100.00 & 100.00 & 0.94 & 97 \\
\hline \multicolumn{9}{|l|}{ Not Consumed } \\
\hline Acartia lilljeborgi & - & - & $\underline{-0.61}$ & - & - & - & - & - \\
\hline Metamysidopsis elongata atlantica & - & - & -0.03 & - & - & - & $\underline{-0.75}$ & - \\
\hline
\end{tabular}

Haemulon sp was not present in autumn and had its higher abundance in spring (Fig. 3). The species fed largely on Mysidopsis coelhoi in spring, $K$. schubarti and $A$. armillatus in winter (Table 7). The species showed avoidance to $M$. elongata atlantica in spring.

I. parvipinnis was not collected in summer and had its higher abundance in autumn and winter (Fig. 3). The species ate Euphausiacea, Mysidopsis tortonesi and $A$. americanus americanus in autumn; $K$ schubarti and $A$. minikoi in winter (Table 8). The species showed avoidance to $M$. elongata atlantica in autumn and winter; and to $A$. minikoi in winter. It has a narrow food spectrum as only 2 to 5 types of items were present in the stomach at any time period.

Table 7. Crustaceans found in stomachs of Haemulon $\mathrm{sp}(\mathrm{N}=26)$ - numerical abundance $(\mathrm{N})$, vacuity index (V.I.), species richness (S.R.), feeding preference (FP), percentage of the total number $(F \%)$ and percentage of total volume $(\mathrm{V} \%)$

\begin{tabular}{|c|c|c|c|c|c|c|c|c|}
\hline \multirow[b]{3}{*}{ Prey item } & \multicolumn{4}{|c|}{ Spring } & \multicolumn{4}{|c|}{ Winter } \\
\hline & \multicolumn{2}{|c|}{ V.I. $=45.46$} & \multicolumn{2}{|c|}{ S.R. $=3$} & \multicolumn{4}{|c|}{ V.I. $=0$ S. R. $=4$} \\
\hline & $\mathrm{F} \%$ & $\vee \%$ & $\mathrm{FP}$ & $\mathrm{N}$ & $\mathrm{F} \%$ & $\vee \%$ & $\mathrm{FP}$ & $N$ \\
\hline \multicolumn{9}{|l|}{ COPEPODA } \\
\hline Cyprideis salebrosa hartmanni & 3.19 & - & 0.03 & 3 & - & - & - & - \\
\hline DECAPODA (larvae) & - & - & - & - & 2.44 & - & 0.01 & 1 \\
\hline \multicolumn{9}{|l|}{ SERGESTIDAE } \\
\hline $\begin{array}{l}\text { Acetes americanus americanus } \\
\text { CARIDEA }\end{array}$ & - & - & - & - & - & - & - & - \\
\hline Alpheus armillatus & - & - & - & - & 7.32 & 60.00 & 0.07 & 3 \\
\hline Ogyrides alphaerostris & 7.45 & 67.31 & 0.07 & 7 & - & - & - & - \\
\hline \multicolumn{9}{|l|}{ MYSIDACEA } \\
\hline Mysidopsis coelhoi & 89.36 & 32.69 & $\underline{0.89}$ & 84 & - & - & - & - \\
\hline \multicolumn{9}{|l|}{ TANAIDACEA } \\
\hline Kalliapseudes schubarti & - & - & - & - & 85.36 & 40.00 & 0.02 & 35 \\
\hline \multicolumn{9}{|l|}{ AMPHIPODA } \\
\hline Corophium acherusicum & - & - & - & - & 4.88 & - & 0.03 & 2 \\
\hline \multicolumn{9}{|l|}{ Not Consumed } \\
\hline Acartia lilljeborgi & - & - & - & - & - & - & - & - \\
\hline Metamysidopsis elongata atlantica & - & - & -0.99 & - & - & - & - & - \\
\hline
\end{tabular}


Table 8. Crustaceans found in stomachs of Isopisthus parvipinis $(\mathrm{N}=62)$ - numerical abundance (N), vacuity index (N.I.), species richness (S.R.), feeding preference (FP), percentage of the total number $(\mathrm{F} \%)$ and percentage of total volume $(\mathrm{N} \%)$

\begin{tabular}{|c|c|c|c|c|c|c|c|c|}
\hline \multirow[b]{3}{*}{ Prey item } & \multicolumn{4}{|c|}{ Autumn } & \multicolumn{4}{|c|}{ Winter } \\
\hline & \multicolumn{2}{|c|}{ V.I. $=19.36$} & \multicolumn{2}{|c|}{ S. R. $=5$} & \multicolumn{2}{|c|}{$\mathrm{V} .1=85.72$} & \multicolumn{2}{|c|}{ S. R. $=2$} \\
\hline & $\mathrm{F} \%$ & $\vee \%$ & FP & $N$ & $\mathrm{~F} \%$ & V\% & $\mathrm{FP}$ & $\mathrm{N}$ \\
\hline \multicolumn{9}{|l|}{ EUPHAUSIACEA } \\
\hline Euphausiacea (not identified) & 50.00 & - & $\underline{0.50}$ & 7 & - & - & - & - \\
\hline \multicolumn{9}{|l|}{ SERGESTIDAE } \\
\hline Acetes americanus americanus & 14.29 & - & 0.14 & 2 & - & - & - & - \\
\hline \multicolumn{9}{|l|}{ CARIDEA } \\
\hline Alpheus armillatus & 7.14 & - & 0.07 & 1 & - & - & - & - \\
\hline \multicolumn{9}{|l|}{ THALASSINIDEA } \\
\hline Callianassidae (not identified) & 7.14 & - & 0.07 & 1 & - & - & - & - \\
\hline \multicolumn{9}{|l|}{ MYSIDACEA } \\
\hline Mysidopsis tortonesi & 21.43 & - & $\underline{0.21}$ & 3 & - & - & - & - \\
\hline \multicolumn{9}{|l|}{ TANAIDACEA } \\
\hline Kalliapseudes schubarti & - & - & - & - & 83.33 & - & $\underline{0.81}$ & 5 \\
\hline \multicolumn{9}{|l|}{ AMPHIPODA } \\
\hline Atylus minikoi & - & - & - & - & 16.66 & - & -0.21 & 1 \\
\hline \multicolumn{9}{|l|}{ Not consumed } \\
\hline Metamysidopsis elongata atlantica & & - & -0.75 & & - & - & -0.27 & - \\
\hline
\end{tabular}

Oligoplites sp was collected throughout the year and in larger numbers during spring and summer (Fig. 3). In spring it ate basically two species of mysids, in summer, the species ingested mainly $A$. americanus americanus, decapod larvae and Lucifer faxoni (Table 9). In autumn it fed basically on $M$. elongata atlantica and $T$. stylifera, and in winter, $M$. elongata atlantica and $L$. fluviatilis. The number of items in the stomach contents of this species varied from 10 in spring to 2 in autumn. The species showed avoidance to $M$. elongata atlantica, $A$. minikoi and $A$. lilljeborgi.

$S$. pachygaster was present throughout the year but it didn't eat crustaceans in summer and presented higher abundance during winter and spring (Fig. 3). The species

Table 9. Crustaceans found in stomachs of Oligoplites sp $(N=84)$ - numerical abundance (N), vacuity index (V.I.), species richness (S.R.), feeding preference (FP), percentage of the total number (F\%) and percentage of total volume (V\%)

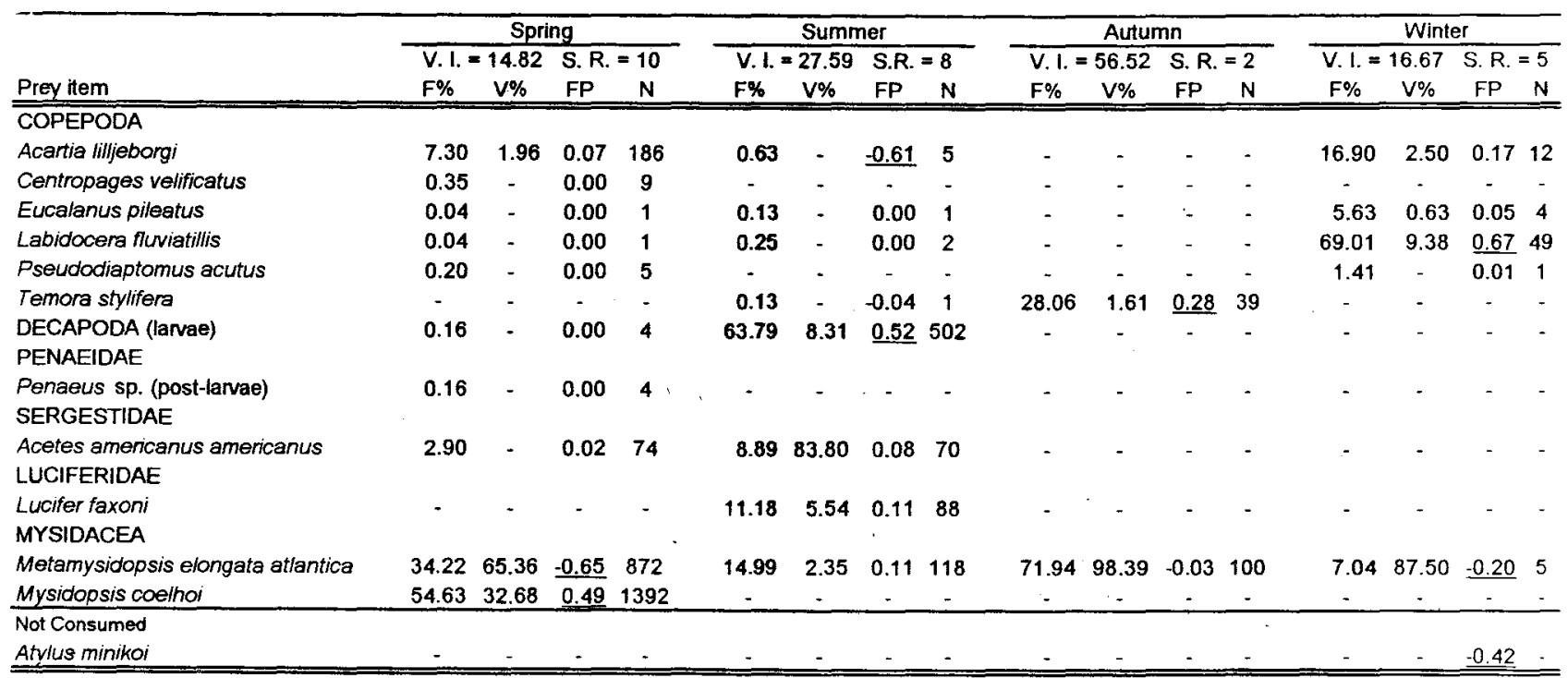


ate, in spring, Callinectes sp and Sphaeromopsis mourei (Table 10). In autumn, it ingested Callinectes sp and $K$. schubarti. During winter, it utilized mainly Parhyale hawaiensis, Ampithoe ramondi and $K$. schubarti. The species showed avoidance to $M$. elongata atlantica in spring, autumn and winter and $A$. minikoi in winter.
To investigate resource partitioning between fish species, food niche overlap was calculated each season from spring to winter, when possible (Table 11). Mean food niche overlap between pairs of fish or group of fish was estimated to be 0.08 . The seasonal food overlap values to determine the interspecific differences in utilization of food

Table 10. Crustaceans found in stomachs of Sphoeroides pachygaster $(\mathrm{N}=21)$ - numerical abundance $(\mathrm{N})$, vacuity index (V.I.), species richness (S.R.), feeding preference (FP), percentage of the total number (F\%) and percentage of total volume (N\%)

\begin{tabular}{|c|c|c|c|c|c|c|c|c|c|c|c|c|}
\hline \multirow[b]{3}{*}{ Prey item } & \multicolumn{4}{|c|}{ Spring } & \multicolumn{4}{|c|}{ Autumn } & \multicolumn{4}{|c|}{ Winter } \\
\hline & \multicolumn{2}{|c|}{ V.I. $=50.00$} & \multicolumn{2}{|c|}{ S. R. $=2$} & \multicolumn{4}{|c|}{ V.I. $=0$ S.R. $=2$} & \multicolumn{4}{|c|}{ V. $1 .=0$ S.R. $=6$} \\
\hline & $\mathrm{F} \%$ & V\% & $\mathrm{FP}$ & $\mathbf{N}$ & $\mathrm{F} \%$ & $\mathrm{~V} \%$ & $\mathrm{FP}$ & $\mathrm{N}$ & $\mathrm{F} \%$ & V\% & FP & $\mathrm{N}$ \\
\hline \multicolumn{13}{|l|}{ BRACHYURA } \\
\hline Callinectes sp. & 50.00 & 67.53 & $\underline{0.50}$ & 1 & 50.00 & 98.04 & $\underline{0.50}$ & 1 & - & - & - & - \\
\hline \multicolumn{13}{|l|}{ MYSIDACEA } \\
\hline \multicolumn{13}{|l|}{ TANAIDACEA } \\
\hline Kalliapseudes schubarti & - & - & - & - & 50.00 & 1.96 & $\underline{0.44}$ & 1 & 13.04 & 3.60 & 0.11 & 3 \\
\hline \multicolumn{13}{|l|}{ ISOPODA } \\
\hline Sphaeromopsis mourei & 50.00 & 32.47 & $\underline{0.50}$ & 1 & - & - & - & - & - & - & - & - \\
\hline \multicolumn{13}{|l|}{ AMPHIPODA } \\
\hline Ampithoe ramondi & - & - & - & - & - & - & - & - & 17.39 & 2.16 & 0.08 & 4 \\
\hline Atylus minikoi & - & - & - & - & - & - & - & - & 8.70 & 1.44 & -0.34 & 2 \\
\hline Hyale sp. & - & - & - & - & - & - & - & - & 4.35 & - & 0.00 & 1 \\
\hline Platorchestia sp. & - & - & - & - & - & - & - & - & 4.35 & - & 0.00 & 1 \\
\hline Parhyale hawaiensis & - & - & - & - & - & - & - & - & 52.17 & 92.81 & $\underline{0.52}$ & 12 \\
\hline \multicolumn{13}{|l|}{ Not Consumed } \\
\hline Metamysidopsis elongata atlantica & - & - & $\underline{-0.99}$ & - & - & - & $-\underline{-0.75}$ & - & - & - & -0.27 & - \\
\hline
\end{tabular}

Table 11. Food - niche overlap $(\alpha)$ between all pairs of species for each season during the period August/1981 to July/1982

\begin{tabular}{lccccc}
\hline \multicolumn{1}{c}{ Species/Group } & Spring & Summer & Autumn & Winter & $\bar{\alpha}$ \\
\hline Group I/Group II & 0.43 & 0.00 & - & 0.00 & 0.140 \\
Group I/Cathorops spixii & 0.00 & 0.00 & - & 0.00 & 0.000 \\
Group I/Eucinostomus sp. & - & 0.00 & - & 0.00 & 0.000 \\
Group I/Haemulon sp. & 0.00 & 0.00 & - & 0.00 & 0.000 \\
Group I//sopisthus parvipinnis & 0.00 & - & - & 0.00 & 0.000 \\
Group I/Oligoplites sp. & 0.44 & $\underline{0.89}$ & - & 0.13 & 0.490 \\
Group I/Sphoeroides pachygaster & 0.00 & - & - & 0.00 & 0.000 \\
Group II/Cathorops spixii & 0.00 & $\underline{0.85}$ & 0.00 & 0.15 & 0.250 \\
Group II/Eucinostomus sp. & - & 0.00 & 0.01 & - & 0.005 \\
Group II/Haemulon sp. & 0.20 & 0.00 & - & 0.47 & 0.330 \\
Group II//sopisthus parvipinnis & - & 0.00 & 0.00 & 0.40 & 0.130 \\
Group II/Oligoplites sp. & 0.05 & - & 0.50 & 0.00 & 0.180 \\
Group II/Sphoeroides pachygaster & 0.00 & - & 0.01 & 0.06 & 0.020 \\
Cathorops spixil/Eucinostomus sp. & - & 0.00 & -0.00 & - & 0.000 \\
Cathorops spixiil/Haemulon sp. & 0.00 & 0.00 & - & 0.58 & 0.520 \\
Cathorops spixill/sopisthus parvipinnis & - & 0.00 & 0.00 & 0.08 & 0.026 \\
Cathorops spixil/Oligoplites sp. & 0.00 & 0.00 & 0.00 & 0.00 & 0.000 \\
Cathorops spixii/Sphoeroides pachygaster & - & 0.00 & 0.00 & 0.05 & 0.016 \\
Eucinostomus sp./Haemulon sp. & - & 0.00 & - & - & 0.000 \\
Eucinostomus sp.//sopisthus parvipinnis & - & - & 0.00 & - & 0.000 \\
Eucinostomus sp./Oligoplites sp. & - & 0.00 & 0.00 & - & 0.000 \\
Eucinostomus sp./Sphoeroides pachygaster & - & - & 0.02 & - & 0.020 \\
Haemulon sp.//sopisthus parvipinnis & 0.00 & - & - & 0.40 & 0.200 \\
Haemulon sp./Oligoplites sp. & 0.32 & 0.00 & - & 0.00 & 0.106 \\
Haemulon sp./Sphoeroides pachygaster & - & 0.00 & - & 0.04 & 0.020 \\
Isopisthus parvipinnis/Oligoplites sp. & 0.00 & - & 0.00 & 0.00 & 0.000 \\
Isopisthus parvipinnis/Sphoeroides pachygaster & 0.00 & - & 0.00 & 0.04 & 0.013 \\
Oligoplites sp./Sphoeroides pachygaster & 0.00 & - & 0.00 & 0.00 & 0.000 \\
mean overlap for fish community & 0.08 & 0.15 & 0.03 & 0.11 & 0.080 \\
\hline \hline
\end{tabular}


resource resulted that the overlap occurred at two situations in summer between $C$. spixii and each species of Group II and between Oligoplites sp and species of Group I. Data of annual food niche overlap between pair of species within Groups I and II are presented on Table 12.

Table 12. Annual food niche overlap $(\alpha)$ between pairs of species within groups

\begin{tabular}{|c|c|c|c|}
\hline Group & Species & $\alpha$ & $\bar{\alpha}$ \\
\hline 1 & $\begin{array}{l}\text { Anchoviella brevirostris } \times \text { Opisthonema oglinum } \\
\text { Anchoviella brevirostris } \times \text { Harengula jaguana }\end{array}$ & $\begin{array}{l}0.68 \\
0.66\end{array}$ & 0.67 \\
\hline II & $\begin{array}{l}\text { Micropogonias furnieri } \times \text { Stellifer stellifer } \\
\text { Micropogonias furnieri } \times \text { Symphurus jenynsii } \\
\text { Symphurus jeninsii } \times \text { Stellifer stellifer }\end{array}$ & $\begin{array}{l}0.88 \\
0.82 \\
0.91\end{array}$ & 0.87 \\
\hline
\end{tabular}

\section{Discussion}

It was considered in our previous work (Wakabara et al., 1993) that the majority of the twelve fish species analysed have their main food source on groups as mysids, copepods, epifaunal crustaceans as amphipods and isopods, infaunal tanaids besides decapod larvae and sergestids, comprising more than $70 \%$ of the diet. Indeed, such organisms could be considered as making part of the suprabenthos (sensu Dauvin et al., 1994). So, here we will treat them as suprabenthic species and that is why only crustaceans items were thoroughly investigated in this paper.

In Arrozal, Cananéia lagoon estuarine region, as in other saltmarsh or seagrass systems, crustacean members of suprabenthos were the major source of available food for small sized fish (Burchmore et al., 1984; Wakabara et al., 1993). Even for continental shelf fishes, in their first year, these small mobile benthic crustaceans are the most important food resource (Sorbe, 1981).

The suprabenthic community of a shallow coastal area has a marked seasonal pattern. This is mainly due to the sequential appearance, high abundance and disappearance of temporary suprabenthic species (Mees \& Hamerlynck, 1992). Even in brackish water parts of estuary where most temporary and migratory suprabenthic species are not able to penetrate, the suprabenthic community, although poor in species, has extremely high densities of mysids and strong temporal variations in abundance of species (Mees et al., 1993). In accordance to the latter, Cananéia suprabenthic community at Arrozal shows a low species diversity, mainly dominated by $M$. elongata atlantica, A. lilljeborgi, $A$. minikoi and decapod larvae and presents strong seasonal variations.

The 12 species of fish analyzed in this study are all carnivorous and have the suprabenthic crustaceans as a primary food resource. From this total, 4 species $(A$. brevirostris, $O$. oglinum, $H$. jaguana and Oligoplites sp) utilized mainly suprabenthic crustaceans with planktonic origin such as copepods and decapod larvae, besides mysids, while the remaining 8 species (S. stellifer, $M$. furnieri, $S$. jenynsii, $C$. spixii, Eucinostomus sp, Haemulon sp, I. parvipinnis and $S$. pachygaster) ate suprabenthic crustaceans with benthic origin such as $M$. cananeia, $K$. schubarti, mysids, $A$. armillatus and $A$. minikoi. As some planktonic originated items were present only in stomach contents of fishes, it is concluded that the sampler used in this study was not efficient for the sampling of planktonic copepods, probably due to inadequate mesh size of the bag.

Seasonal variation in total abundance is a common feature in dynamic ecosystems such as estuaries. The local fish assemblage showed seasonal fluctuation. The 12 fish species appeared in higher abundance in different seasons corresponding probably to different recruitment periods into the system. According to Subrahmanyan and Coultas (1980) the staggered seasonal occurrence of species may be in concordance with the local availability of food resources. In the present study the overall number of fish, due mainly to fishes from Groups I and II, increased in the spring and winter with the rising of the suprabenthic crustacean numbers, due mainly to the tremendous rise of $M$. elongata atlantica numbers comprising $99 \%$ of the total fauna. This fact justified the almost uniform negative feeding preference values for $M$. elongata atlantica exhibited by most of the fish species considered, since they should have to consume strictly this mysids to provide feeding preference positive values.

The seasonal changes of the food supply is clearly reflected in the observed seasonal variations in the diet of fishes from Group I and II. In relation to feeding preference, species from Groups I and II ( $A$. brevirostris, $O$. oglinum, $H$. jaguana, S. stellifer, $M$. furnieri and S. jenynsii) were considered opportunistic feeders, since the relative abundance of the more important crustacean food resource in the environment was similar to their relative abundance in stomachs. The other six species, not grouped, were considered selective feeders, since they ingested the crustaceans in proportionally greater numbers than occurred in the environment. These concepts of opportunistic and selective feeders are based on ideas discussed in Sagar and Eldon (1983) and Jacksic (1989). 
The low value, 0.08 of the mean total overlap for all the fish community, indicates that each fish predator or group of fishes have an almost completely distinct food niche, avoiding in this manner food competition.

The same cannot be said for the species within Group I and Group II. Group I species ( $A$. brevirostris, $H$. jaguana and $O$. oglinum) had a significant mean food overlap (0.67) revealing that they use similar food items. Species of Group II (M. furnieri, S. jenynsii and S. stellifer) had also a high mean food overlap ( 0.87$)$. When cohabiting species are similar in food preference and habitat, the question arises as to whether they are in competition defined as the demand of more than one organism for the same resource. According to Mathur (1977) two species exploring a similar niche may coexist without competition if the food resource is temporarily superabundant. This may be true for the fish species in Cananéia estuary.

The significant overlap found in summer seems to have 2 different explanations; between species with benthic originated suprabenthic food resource, the increased food overlap may be due to the diminishing food supply in summer as Thorman (1982) found in estuarine region. He interpreted this raised overlap as increasing competition for food in summer. This would be the case of $C$. spixii and species of Group II. In relation to the summer overlap between Oligoplites sp and species of Group I, species with a planktonic originated suprabenthic food resource, it may indicate superabundance of food resource as this is the time of density peaks of planktonic forms such as some copepods, sergestids and decapod larvac highly ingested by these fish species.

\section{Acknowledgements}

We thank the following specialists: Ana Maria Setubal Pires Vanin (Isopoda and Tanaidacea), Antonio Frederico Campaner (Copepoda), Motonaga Iwai (Peneaidae) and Gustavo A. S. Melo (Brachyura, Caridea and Thalassinidea) for identification of Crustacean species.

\section{References}

Burchmore, J. J.; Pollard, D. A. \& Bell, J. D. 1984. Community structure and trophic relationships of the fish fauna of an estuarine Posidonia australis seagrass habitat in Port Hacking, New South Wales. Aquat. Bot., 18:71-87.
Chao, L. N. \& Musick, J. A. 1977. Life history, feeding habits, and functional morphology of juvenile sciaenid fishes in the York River estuary, Virginia. Fish. Bull., 75(4):657-702.

Davis, I. C. 1973. Statistic and data analysis in geology with FORTRAN programs by Robert J. Sampson. New York, John Wiley \& Sons. 550p.

Dauvin, J. C.; Iglesias, A. \& Lorgeré, J. C. 1994. Circalittoral suprabenthic coarse sand community from the Western English Channel. J. mar. biol. Ass. U.K., 74:543-556.

Evans, S. 1984. Energy budgets and predation impact of dominant epibenthic carnivores on a shallow soft bottom community at the Swedish west coast. Estuar. coast. Shelf. Sci., 18:651-672.

Jacksic, F. M. 1989. Opportunist, selective, and other often- confused terms in the predation literature. Revta. chil. hist. nat., 62:7-8.

Jackson, J. B. C. 1972. The ecology of the molluscs of Thalassia communities, Jamaica, West Indians. II. Moluscan population variability along an environment stress gradient. Mar. Biol., 14(4):304-337.

Kohler, C. \& Ney, J. J. 1982. A comparison of methods for quantitative analysis of feeding selection of fishes. Environ. Biol. Fishes, 7(4):363-368.

Mathur, D. 1977. Food habits and competitive relationships of the bandfin shiner in Halawakee Creek, Alabama. Am. Midl. Naturalist, 97:89-100.

Mees, J. \& Hamerlynck, O. 1992. Spatial community structure of the permanent hyperbenthos of the Schelde-estuary and the adjacent coastal waters. Neth. J. Sea Res., 29:357-370.

Mees, J.; Dewicke, A. \& Hamerlynck, O. 1993. Seasonal composition and spatial distribution of hyperbenthic communities along estuarine gradients in the westernchelde. Neth. J. Aquatic. Ecol., 27(2-4):359-376.

Pihl, L. 1985. Food selection and consumption of mobile epibenthic fauna in shallow marine arcas. Mar. Ecol. - Prog. Ser., 22:169-179. 
Sagar, P. M. \& Eldon, G. A. 1983. Food and fecding of small fish in the Rakaia River, New Zealand. N. Z. Jl mar. Freshwat. Res., 17:213- 226.

Schoener, T. W. 1970. Nonsynchronous spatial overlap of lizards in patchy habitats. Ecology, 51(1/3):408-418.

Sibert, J. R. 1981. Intertidal hyperbenthic populations in the Nanaimo estuary. Mar. Biol., 64:259-265.

Sokal, R. R. \& Sneath, P. H. A. 1973. Principals of numerical taxonomy. San Francisco, W.H. Freeman. 359p.

Sorbe, J. C. 1981. Rôle du benthos dans le régime alimentaire des poissons démersaux du secteur sud Gascogne. Kieler Meeresforsch. Sonderh., 5:479-489.

Strauss, R. E. 1979. Reability estimates for Ivlev's electivity index, the forage ratio, and a proposed linear index of food selection. Trans. Am. Fish. Soc., 108:344-352.

Subrahmanyam, C. B. \& Coultas, C. L. 1980. Studies on the animal communities in two North Florida saltmarshes. III. Seasonal fluctuations of fish and macroinvertebrates. Bull. mar. Sci., 30(4):790-818.

Thorman, S. 1982. Niche dynamics and resource partitioning in a fish guild inhabiting a shallow estuary of the Swedish West Coast. Oikos, 39:32-39.
Thorman, S. \& Wiederholm, A. M. 1983. Scasonal occurrence and food resource use of an assemblage of nearshore fish species in the Bothnian Sea, Sweden. Mar. Ecol.-Prog. Ser., 10:223-229.

Thorman, S. \& Wiederholm, A. M. 1986. Food, habitat and time niches in a coastal fish species assemblage in a brackish water Bay in the Bothinian Sea, Sweden. J. expl. mar. Biol. Ecol., 95:67- 86.

Wakabara, Y.; Tararam, A. S. \& Flynn, M. N. 1993. Importance of the macrofauna for the feeding of young fish species from infralittoral of Arrozal-Cananéia lagoon estuarine region (25 $\left.02^{\prime} \mathrm{S}-47^{\circ} 56^{\prime} \mathrm{W}\right)$ - Brazil. Bolm Inst. oceanogr., S Paulo, 41(1/2):39-52.

Zander, C.D. 1979. On the biology and food of small-sized fish from the North and Baltic Sea areas. II. Investigation of a shallow stony Ground off Mon, Denmark. Ophclia, 18(2):179-190.

Zaret, T. M. \& Rand, A. S. 1971. Competition in tropical stream fishes: support for the competitive exclusions principle. Ecology, 52(2):336-342.

(Manuscript received 11 June 1996; revised 11 August 1996; accepted 16 October 1996) 